\title{
Serum Levels of Growth Factors in Alcohol-dependent Patients according to Comorbid Depressive Symptoms
}

\author{
Changwoo Han ${ }^{1}$, Donghyun Ahn², Woong Hahm³, Junghyun Nam², Yongchon Park ${ }^{2}$, Seulgi Lim ${ }^{4}$, Dai-Jin Kim ${ }^{4}$ \\ 'Department of Psychiatry, Korea University Ansan Hospital, Korea University College of Medicine, Ansan, ${ }^{2}$ Department of Neuropsychiatry, \\ Hanyang University College of Medicine and Institute of Mental Health, Seoul, ${ }^{3}$ Department of Neuropsychiatry, Keyo Hospital, Uiwang, \\ ${ }^{4}$ Department of Psychiatry, Seoul St. Mary's Hospital, College of Medicine, The Catholic University of Korea, Seoul, Korea
}

\begin{abstract}
Objective: This study aims to reveal the relationship of depression with growth factors such as brain-derived neurotrophic factor (BDNF), nerve growth factor (NGF), and insulin-like growth factor-1 (IGF-1) in inpatients diagnosed with alcohol dependence, and to identify candidate growth factors as biological markers to indicate the comorbid of alcohol dependence and depression.

Methods: This study examined demographic factors in 45 alcohol-dependent patients. The ADS (Korean version of the Alcohol Dependence Scale) and BDI (Korean version of Beck's Depression Inventory) were used. BDNF, NGF, and IGF-1 were measured through ELISA.

Results: The average drinking quantity and the ADS score were significantly more severe in alcohol-dependent patients with depression than in those without depression. Linearly comparing BDNF, NGF, and IGF-1 with BDI values, IGF-1 was the growth factor significantly correlated with BDI scores. BDI scores were significantly correlated with ADS scores. IGF-1 was significantly higher in alcohol-dependent patients with depression. Alcohol-dependent patients with depression had greater alcohol use and more severe ADS scores. BDNF and NGF showed no significant difference between alcohol-dependent patients with and without depression, but IGF-1 was significantly higher in those with than in those without depression.

Conclusion: IGF-1 was found to be associated with depression in alcohol-dependent patients, suggesting that IGF-1 in alcohol-dependent patients could be an important biomarker to indicate whether alcohol-dependence is accompanied by depression.

KEY WORDS: Alcohol dependence; Depression; Growth factor; Insulin-like growth factor-1.
\end{abstract}

\section{INTRODUCTION}

Alcohol use disorder is known to have a strong relationship with depression. Alcohol use disorder patients complain of depressive symptoms frequently, and alcohol dependence is accompanied by depression in many cases. ${ }^{1-3)}$

Combined alcohol dependence and depression is likely to lead to more problems than either single disease, such as a higher risk of committing suicide. ${ }^{4)}$ Depressed patients with a medical history of alcoholism tend to be highly aggressive and impulsive and to have a personal history of

Received: May 19, 2015/Revised: June 8, 2015

Accepted: June 9, 2015

Address for correspondence: Dai-Jin Kim, MD, PhD Department of Psychiatry, Seoul St. Mary's Hospital, College of Medicine, The Catholic University of Korea, 222 Banpo-daero, Seocho-gu, Seoul 137-701, Korea

Tel: +82-2-2258-608, Fax: +82-2-594-3870

Changwoo Han's current affiliation is "Department of Psychiatry, Eulji University Gangnam Eulji Hospital, Seoul, Korea." E-mail:kdj922@catholic.ac.kr attempted suicide, and their scores on the suicide rate scale, which includes suicidal ideation and attempted suicide, are higher than for depressed patients without alcohol use. ${ }^{5,6)}$

To reveal causality effects present in the comorbidity of alcohol use disorder and depression, such studies have presented various candidate factors to check for the coexistence of the two diseases. In particular, one study reported that platelet monoamine oxidase was measured as low in alcohol-dependent patients who had severe alcohol symptoms accompanied by depressive symptoms. ${ }^{7)}$ Another study showed that the activity of adenylyl cyclase can be a biological marker in patients with both depression and substance use disorder. ${ }^{8)}$

But a biological marker to indicate whether depressive symptoms are present in alcohol-dependent patients has not yet been discovered. To find such markers, recent studies have been carried out on growth factors.

A growth factor is a composite that is organized with

(ㄷ) This is an Open-Access article distributed under the terms of the Creative Commons Attribution Non-Commercial License (http://creativecommons.org/licenses/by-nc/4.0) which permits unrestricted non-commercial use, distribution, and reproduction in any medium, provided the original work is properly cited. 
various polypeptides and involved in the activity, differentiation, and survival of cells. ${ }^{9}$ Previous studies have revealed that growth factor is associated with various mental diseases, including depression and schizophrenia. ${ }^{10,11)}$ In particular, studies of the association of depression with growth factor showed that brain-derived neurotrophic factor (BDNF) was lower in depressed patients than in normal controls. ${ }^{12)}$ Showing the relationship between depression and nerve growth factor (NGF), the value of NGF was reduced in depressed patients. Insulin-like growth factor-1 (IGF-1) is also reported to have relevance with depression. IGF-1 was higher in depressed patients than in general controls. ${ }^{13,14)}$ Therefore, various existing studies raise the possibility that such growth factors as BDNF, NGF, and IGF-1 are biological markers for depression.

Studies have also investigated the association of alcohol use disorder with growth factor. In these studies, BDNF was low in alcohol-dependent patients and NGF was measured as low in alcohol-dependent patients. ${ }^{15,16)}$

The existing studies additionally demonstrated that insulin has also been shown to be associated with alcohol dependence, but there was no significant association of IGF-1 with alcohol dependence. ${ }^{17,18)}$ IGF-1 was only associated with cravings for drinking in alcohol dependence.

Such existing studies raise the possibility that growth factor is biological marker for either single disease: depression or alcohol use disorder. However, we lack studies of candidate factors to indicate whether depression co-occurs with alcohol-dependence.

Therefore, this study investigated various clinical characteristics of alcohol-dependent patients, implementing alcohol dependence and depression scales, and variations in severity of depressive symptoms in alcohol-dependent patients. Moreover, to investigate growth factor as a biological marker of alcohol dependence co-occurring with depression, representative growth factors BDNF, NGF, and IGF-1 were measured to reveal the difference in growth factor depending on the severity of depressive symptoms in alcohol-dependent patients. This was done to meet the study aim to investigate whether measuring growth factor was clinically significant in alcohol-dependent patients with depression.

\section{METHODS}

\section{Subjects}

The study participants were 45 hospitalized patients from the alcohol center of a mental hospital located in Gyeonggi-do, Korea. They were patients diagnosed with alcohol dependence according to the Diagnostic and Statistical Manual of Mental Disorders 4th edition (DSM-IV). The patients were interviewed regarding their history of alcohol (e.g., duration of problem drinking, family history and withdrawal symptoms). The participant's ages ranged in age from 37 to 62 years. The researchers checked up their other mental disorders and examined the mental status of the patients. This study excluded individuals with mental diseases other than depression, other drug abuse or psychopathologies, or severe head injuries. Participants had been prohibited from drinking for at least one week prior to hospitalization. However, individuals who were still experiencing withdrawal symptoms were excluded from the experimental group because differences in partial growth factors have been observed in the presence of withdrawal symptoms of alcohol. ${ }^{17,19)}$

Three further individuals were excluded from the original 48 participants. One of them showed continuous hand tremor symptoms after hospitalization (i.e., a withdrawal symptom of alcohol), and two responded to some items of

Table 1. Demographic and clinical characteristics of alcohol-dependent patients with/without depression ( $\mathrm{n}=45$ )

\begin{tabular}{|c|c|c|c|c|}
\hline Variable & $\begin{array}{c}\text { A without D } \\
(B D I<21 ; n=24)\end{array}$ & $\begin{array}{c}\text { A with } D \\
(B D I \geq 21 ; n=21)\end{array}$ & $\dagger$ & $p$ value \\
\hline Age $(y r)$ & 52.21 & 50.01 & 1.159 & 0.253 \\
\hline Education (yr) & 15.84 & 19.17 & -0.937 & 0.354 \\
\hline Onset age of drinking alcohol (yr) & 21.41 & 23.14 & -0.797 & 0.43 \\
\hline Frequency of alcohol consumption (per week) & 3.761 & 4.632 & -1.303 & 0.2 \\
\hline Daily amount of alcohol consumed & 2.217 & 5 & -2.642 & $0.012^{*}$ \\
\hline ADS-K & 18.541 & 26.142 & -2.414 & $0.020^{*}$ \\
\hline BDNF (pg/ml) & $3,081.78$ & $2,953.886$ & 0.381 & 0.705 \\
\hline NGF $(\mathrm{pg} / \mathrm{ml})$ & 26.803 & 27.547 & -0.243 & 0.809 \\
\hline IGF-1 (pg/ml) & 29.477 & 39.692 & -2.876 & $0.006^{* *}$ \\
\hline
\end{tabular}

A without $D$, alcohol-dependent patients without depression; A with $D$, alcohol-dependent patients with depression; ADS-K, Korean version of Alcohol Dependence Scale; BDI, Beck's Depression Inventory; $t$, independent t-test: BDNF, brain-derived neurotrophic factor: NGF, nerve growth factor; IGF-1, insulin-like growth factor-1.

${ }^{*} p<0.05$, ${ }^{* *} p<0.01$. 
the questionnaire inconsistently with other observations.

The demographic and clinical characteristics of research participants are presented in Table 1. This study was carried out after approval by the screening committee for clinical tests in Seoul St. Mary's Hospital. Because this study conducted in the men only ward, all research participants were males; the purpose of this study was explained to them by the researchers, and they signed consent forms to participate in this study voluntarily. All procedures were carried out with the approval of institutional review board of the Catholic University of Korea (C11QISI0569).

\section{Measurements}

Survey questions measured demographic variables of the participants, including age, education, onset age of drinking alcohol, average frequency of drinking, and average drinking quantity. To evaluate alcohol dependence severity, we employed the Korean version of the Alcohol Dependence Scale (ADS), which consists of 25 questions developed by Skinner and Allen through factor analysis. This scale evaluates compulsive drinking, drinking problem behavior, and alcohol withdrawal symptoms, and the form used was standardized by Lee et $_{\text {al }}{ }^{20)}$ in Korea.

The Korean version of the Beck Depression Inventory (BDI) was used to evaluate depressive symptoms. The BDI is a self-report measure widely used for examination of depressed patients and is useful for evaluating the degree and patterns of depressive symptoms. It is composed of 21 questions addressing emotion, recognition, motif, physical, and other symptoms: each question is scored from 0 to 3 points, and the maximum total score is 63 points. The research by Han et al. ${ }^{21)}$ in Korea that has standardized this inventory has been cited in many published studies.

The questionnaire was distributed to all participants simultaneously, and a psychiatrist instructed them about how to respond to the questionnaire.

\section{Blood Analysis}

Blood was collected by one nurse from experimental participants between 10:00 a.m. and 11:00 p.m. All participants had breakfast at 8:00 a.m. and took a rest or participated in the educational programs provided in the ward after breakfast. They did not exercise or work in ways that caused exhaustion of physical strength before their blood collection.

Each blood sample of $10 \mathrm{ml}$ was collected in an EDTA-coated tube and then immediately centrifuged, from which blood plasma was collected and then stored at $80^{\circ} \mathrm{C}$ below zero until it could be analyzed.

BDNF was measured using the Human Brain-Derived Protein Multiplex Immunoassay Kit (the coefficient of variation-inter-assay $[\% \mathrm{CV}],<15$; intra-assay $[\% \mathrm{CV}]$, $<10$ ), and NGF was measured using the Human Adipokine Magnetic Bead Panel 2 - Endocrine Multiplex Assay (the coefficient of variation-inter-assay [\%CV], $<11$; intra-assay [\%CV], <4). To measure IGF-1, the IGF-1 ELISA Kit (the coefficient of variation-inter-assay $[\% \mathrm{CV}],<15$; intra-assay $[\% \mathrm{CV}],<10)$ was used. All products were bought from Millipore (Billerica, MA, USA), and the experiment was carried out according to the manufacturer's standard guidelines. The concentration of each plate was corrected through standard curve and dilution factors.

\section{Statistical Analysis}

To distinguish the alcohol-dependent patients with depression from those without depression, the two groups were divided on the basis of BDI scores of at least 21 . This criterion is based on the fact that a score of 21 is considered to be the most appropriate division point for determining the presence of depression in Korea, unlike in other countries, as established through the validation study of the BDI conducted by Han et al., ${ }^{21)}$ and, in general, Korean studies are using this value as the standard criterion. $^{21)}$

We used independent sample $t$-tests to verify (depressed versus non-depressed) group differences in demographic variables, such as age, education, onset age of drinking alcohol, average frequency of drinking, and average drinking quantity. Also, an independent sample $t$-test was used to examine group difference in ADS scores.

We performed correlational analysis to determine bivariate correlations among age, average quantity of drinking, BDI scores, ADS scores, and growth factors BDNF, NGF, and IGF-1 in the entire participant sample.

We used independent sample $t$-tests to analyze the group differences in BDNF, NGF, and IGF-1, i.e., in alcohol-dependent patients with depression versus those without them.

We conducted the statistical analysis using Statistical Package for Social Sciences (SPSS) software version 16.0 for Windows (SPSS Inc., Chicago, IL, USA), and we set the significance level for all analyses at $p<0.05$.

\section{RESULTS}

The group of alcohol-dependent patients without de- 
pression consisted of 24 patients who scored less than 21 on the BDI, and the group of alcohol-dependent patients with depression consisted of 21 patients who scored more 21 on the BDI.

As shown in Table 1, there were no differences between the two groups in terms of age, education, onset age of drinking alcohol, and average frequency of drinking. However, the average drinking quantity was significantly higher, at a level of 5 bottles (on the basis of soju: a distilled beverage native to Korea), in alcohol-dependent patients with depression than in those without depression, at a level of 2.21 bottles (on the basis of soju) $(\mathrm{t}=-2.642$, $p=0.012$ ). The ADS scores, which reflect the severity of alcohol dependence, were significantly higher (group mean=26.14) in alcohol-dependent patients with depression than in those without depression (group mean=18.54) $(\mathrm{t}=-2.414, p=0.020)$, as shown in Table 1 .

The correlational analysis to determine the relationships among BDI score, ADS score, and the examined growth factors in the entire experimental sample showed that BDI score was significantly correlated with ADS score $(\mathrm{r}=0.430, p=0.004)$ (Table 2$)$. Also, the growth factor IGF-1 was significantly correlated with BDI $(\mathrm{r}=0.307$,

Table 2. Correlations among serum IGF-1 level, BDI score, and ADS-K score

\begin{tabular}{lccc}
\hline & IGF-1 & BDI & ADS-K \\
\hline IGF-1 & 1 & & \\
BDI & $0.04^{*}$ & 1 & \\
ADS-K & 0.762 & $0.004^{* *}$ & 1 \\
\hline
\end{tabular}

IGF-1, insulin-like growth factor-1; BDI, Beck's Depression Inventory; ADS-K, Korean version of Alcohol Dependence Scale. ${ }^{*} p<0.05,{ }^{* *} p<0.01$.

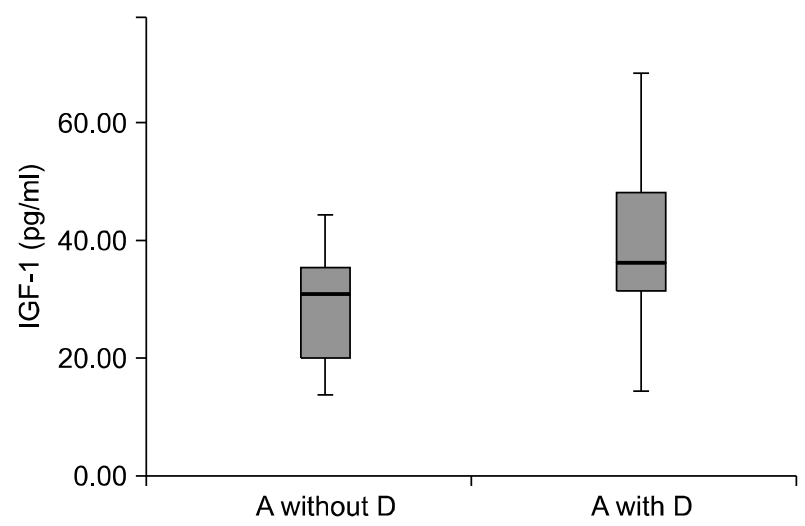

Fig. 1. Comparison of serum insulin-like growth factor-1 (IGF-1) levels between alcohol-dependent patients with/without depression. A without D, alcohol-dependent patients without depression; A with $\mathrm{D}$, alcohol-dependent patients with depression. $p=0.04$ ) (Table 2). However, growth factors BDNF and NGF had no significant association with BDI.

Comparing the BDNF, NGF, and IGF-1 values in the two (depressed versus non-depressed) groups, IGF-1 was significantly higher in alcohol-dependent patients with depression than in those without depression $(\mathrm{t}=2.876$, $p=0.006$ ) (Table 1, Fig. 1), whereas BDNF and NGF showed no significant group difference (Figs. 2, 3).

\section{DISCUSSION}

This study was carried out to investigate differences in severity of alcohol-related symptoms in depressed versus non-depressed alcohol-dependent patients and to determine a biological marker to indicate the coexistence of depression with alcohol dependence.

The results showed that BDI score was associated with

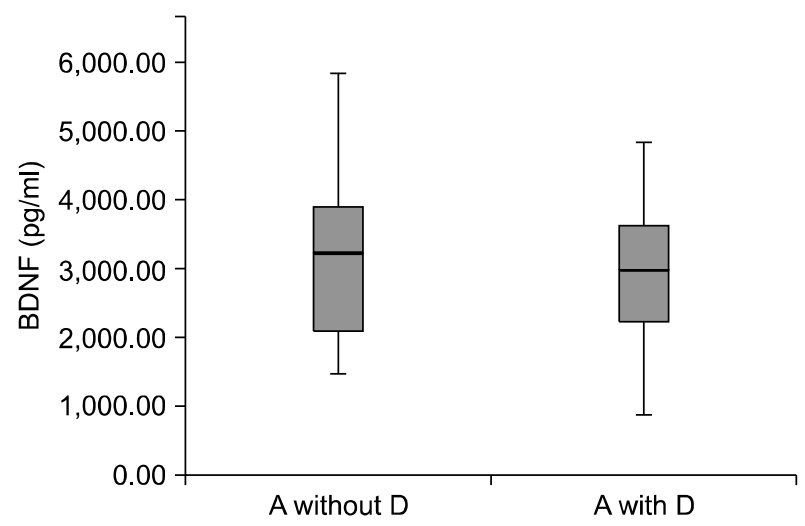

Fig. 2. Comparison of serum brain-derived neurotrophic factor (BDNF) levels between alcohol-dependent patients with/without depression.

A without $D$, alcohol-dependent patients without depression; $A$ with D, alcohol dependent patient with depression.

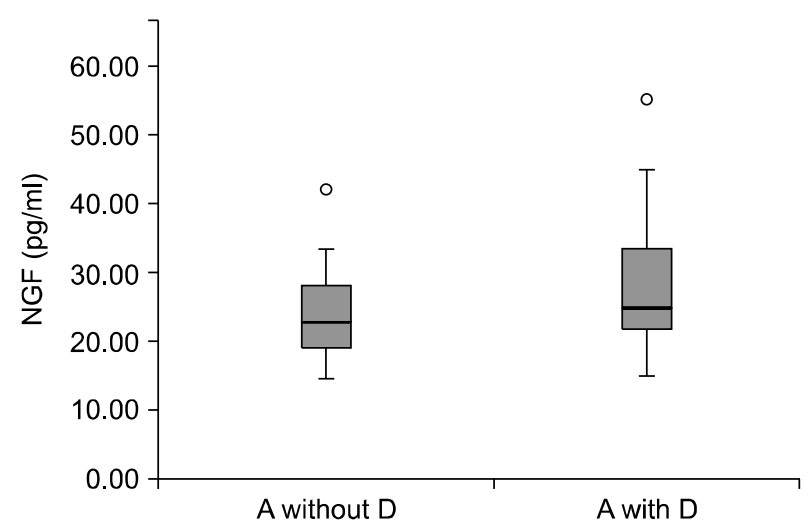

Fig. 3. Comparison of serum nerve growth factor (NGF) levels between alcohol-dependent patients with/without depression. A without D, alcohol-dependent patients without depression; A with $\mathrm{D}$, alcohol-dependent patients with depression. 
ADS score, and alcohol use disorder problems were more severe in depressed than in non-depressed alcohol-dependent patients. Alcohol-dependent patients who were also depressed, compared to those who were not, had much greater average drinking quantity and much higher ADS scores for severity of alcohol use disorder. This clarifies the existing theory that, when depression co-occurs with alcohol abuse, the alcohol dependence severity was generally higher. ${ }^{22)}$

In this study analyzing the difference in growth factors between alcohol-dependent patients with versus without depression, BDNF and NGF showed no significant group difference, whereas IGF-1 was significantly higher in those with depression. And in the entire patient sample, IGF-1 had a significant correlation with BDI score.

In previous research, BDNF has been measured as lower in depressed patients than in normal controls and, likewise, measured as lower in alcohol-dependent patients than in normal controls. ${ }^{10,16)}$ However, studies in which depressed patients were divided into those with versus those without alcohol dependence have reported no significant group difference in BDNF. ${ }^{23)}$ In this study, BDNF did not show a statistically significant tendency to be lower in alcohol-dependent patients with depression than in those without depression. However, BDNF is the well-known growth factor about the alcohol dependence and depression. More studies for the BDNF in alcohol-dependence accompanied by depression are needed.

In studies of NGF and mental diseases, NGF has been measured as lower in older depressed patients. ${ }^{24)}$ NGF has also been measured as lower in alcohol-dependent patients than in normal controls. ${ }^{15)}$ Of course, no research has yet investigated changes in NGF in comorbid alcohol dependence and depression. In this study, NGF was slightly higher in alcohol-dependent patients with depression than in those without depression, but the difference was not significant.

It is noteworthy in this study that IGF-1 was significantly higher in alcohol-dependent patients with depression than in those without depression. This indicates that IGF-1 is likely to reflect depression because IGF-1 was higher when alcohol-dependence was accompanied by depression than when it was not. Similarly, IGF-1 was higher in patients with depression than in normal controls without depression. ${ }^{13)}$ In this study, plasma IGF-1 level was found to be significantly higher in depressed patients, while there was no difference in growth hormone between both groups. And the concentration of IGF-1 was increased in depressive patients after treatment.

IGF-1 is known to be affected by the increased secre- tion of growth hormone. ${ }^{14)}$ Previous studies have reported that the secretion of growth hormone is increased by upregulation of growth-hormone-releasing hormone in depressed patients. ${ }^{25)}$ It is currently known that an increased secretion of growth hormone activates the synthesis and activity of IGF-1. Therefore, changes in the values of IGF-1 in serum can indicate an imbalance of the hypothalamus-pituitary gland-adrenal system in depression.

However, no precise information has been reported on the association of alcohol dependence with IGF-1. Some research has reported an association of insulin or IGF-1 with addiction disorder, but other research has reported no association of IGF-1 with alcohol dependence. ${ }^{17,18)}$ In the present study, we found no significant association between IGF-1 and alcohol dependence.

Therefore, the fact that IGF-1 is significantly higher in alcohol-dependent patients with depression than in those without may result from a specialized reaction of IGF-1 against depressive symptoms, unrelated to alcohol dependence. In other words, IGF-1 appears to be a biological marker that reflects depression levels in alcohol-dependent patients, which is consistent with the findings of earlier studies that IGF-1 reflects depressive symptoms biologically in depressed patients.

There are things to consider in treating alcohol-dependent patients with depression versus patients with alcohol dependence alone. Patients with both diseases tend to present more severe clinical symptoms than do single-disease patients with either depression or alcohol dependence, and in particular, they appeared to have a higher risk of committing suicide or having significantly degraded self-management ability. ${ }^{5,6)}$ In addition, in most cases of alcohol withdrawal, depressive symptoms may be concealed or become more severe, and with the accompanying medical diseases, there is a higher risk of mentally and physically deteriorated health. Alcohol-dependent patients with depression had a poorer prognosis than those without depression. ${ }^{26)}$ Therefore, care and observation to diagnose the coexistence of the two diseases are essential. To this end, IGF-1 shows promise as a clinically important tool for identifying coexisting alcohol dependence and depression.

This study explored the clinical characteristics of alcohol-dependent patients with depression and their biological markers. However, this study has some limitations.

First, the experimental sample is small. In addition, our research participants were only male patients, and no results were drawn from female alcohol-dependent patients.

Second, this study could not control changes that depend on individual conditions, such as the patient's dinner, 
hunger state, and physical symptoms during blood gathering. Because growth factor may be affected by such physiological differences, future studies are needed under consistent environmental conditions in these regards.

Finally, this study divided the alcohol-dependent patients into those with and those without depression on the basis of BDI scores of at least 21 as the criterion for classifying patients as depressed. This method has been used in the existing research by Han et al. ${ }^{21)}$ because the BDI criterion (9 points) used in other countries is not appropriate for Koreans. For greater clarification, future studies are needed with larger samples and using more refined BDI categories.

\section{Acknowledgments}

This study was supported by a grant from the Korean Health Technology R\&D Project, Ministry of Health and Welfare, South Korea (HI11C1332).

\section{REFERENCES}

1. Kessler RC, Nelson CB, McGonagle KA, Edlund MJ, Frank RG, Leaf PJ. The epidemiology of co-occurring addictive and mental disorders: implications for prevention and service utilization. Am J Orthopsychiatry 1996;66:17-31.

2. Kessler RC, Crum RM, Warner LA, Nelson CB, Schulenberg $\mathrm{J}$, Anthony JC. Lifetime co-occurrence of DSM-III-R alcohol abuse and dependence with other psychiatric disorders in the National Comorbidity Survey. Arch Gen Psychiatry 1997;54:313-321.

3. Regier DA, Farmer ME, Rae DS, Locke BZ, Keith SJ, Judd $\mathrm{LL}$, et al. Comorbidity of mental disorders with alcohol and other drug abuse. Results from the Epidemiologic Catchment Area (ECA) Study. JAMA 1990;264:2511-2518.

4. Cornelius JR, Salloum IM, Mezzich J, Cornelius MD, Fabrega H Jr, Ehler JG, et al. Disproportionate suicidality in patients with comorbid major depression and alcoholism. Am J Psychiatry 1995:152:358-364.

5. Davis LL, Rush JA, Wisniewski SR, Rice K, Cassano P, Jewell ME, et al. Substance use disorder comorbidity in major depressive disorder: an exploratory analysis of the Sequenced Treatment Alternatives to Relieve Depression cohort. Compr Psychiatry 2005;46:81-89.

6. Sher L, Oquendo MA, Galfalvy HC, Grunebaum MF, Burke $\mathrm{AK}$, Zalsman $\mathrm{G}$, et al. The relationship of aggression to suicidal behavior in depressed patients with a history of alcoholism. Addict Behav 2005;30:1144-1153.

7. von Knorring AL, Bohman M, von Knorring L, Oreland L. Platelet MAO activity as a biological marker in subgroups of alcoholism. Acta Psychiatr Scand 1985;72:51-58.

8. Hines LM, Tabakoff B; WHO/ISBRA Study on State and Trait Markers of Alcohol Use and Dependence Investigators. Platelet adenylyl cyclase activity: a biological marker for major depression and recent drug use. Biol Psychiatry 2005;58:955-962.

9. McAllister AK, Katz LC, Lo DC. Neurotrophins and synaptic plasticity. Annu Rev Neurosci 1999;22:295-318.
10. Angelucci F, Brenè S, Mathé AA. BDNF in schizophrenia, depression and corresponding animal models. Mol Psychiatry 2005; 10:345-352.

11. Siegel GJ, Chauhan NB. Neurotrophic factors in Alzheimer's and Parkinson's disease brain. Brain Res Brain Res Rev 2000;33:199-227.

12. Karege F, Perret G, Bondolfi G, Schwald M, Bertschy G, Aubry JM. Decreased serum brain-derived neurotrophic factor levels in major depressed patients. Psychiatry Res 2002; 109:143-148.

13. Deuschle M, Blum WF, Strasburger CJ, Schweiger U, Weber $\mathrm{B}$, Körner $\mathrm{A}$, et al. Insulin-like growth factor-I (IGF-I) plasma concentrations are increased in depressed patients. Psychoneuroendocrinology 1997;22:493-503.

14. Tannenbaum GS, Guyda HJ, Posner BI. Insulin-like growth factors: a role in growth hormone negative feedback and body weight regulation via brain. Science 1983;220:77-79.

15. Yoon SJ, Roh S, Lee H, Lee JY, Lee BH, Kim YK, et al. Possible role of nerve growth factor in the pathogenesis of alcohol dependence. Alcohol Clin Exp Res 2006;30:1060-1065.

16. Joe KH, Kim YK, Kim TS, Roh SW, Choi SW, Kim YB, et al. Decreased plasma brain-derived neurotrophic factor levels in patients with alcohol dependence. Alcohol Clin Exp Res 2007;31:1833-1838.

17. Janak PH, Wolf FW, Heberlein U, Pandey SC, Logrip ML, Ron D. BIG news in alcohol addiction: new findings on growth factor pathways BDNF, insulin, and GDNF. Alcohol Clin Exp Res 2006;30:214-221.

18. Leggio L, Ferrulli A, Malandrino N, Miceli A, Capristo E, Gasbarrini $\mathrm{G}$, et al. Insulin but not insulin growth factor-1 correlates with craving in currently drinking alcoholdependent patients. Alcohol Clin Exp Res 2008;32:450-458.

19. Costa MA, Girard M, Dalmay F, Malauzat D. Brain-derived neurotrophic factor serum levels in alcohol-dependent subjects 6 months after alcohol withdrawal. Alcohol Clin Exp Res 2011;35:1966-1973.

20. Lee DK, Shin JK, Yun SM, Byun WT. A reliability and validity study of the Korean version of the alcohol dependence scale in alcoholics. J Korean Acad Addict Psychiatry 2000;4:30-37.

21. Han HM, Yeom TH, Shin YW, Kim KH, Yun DJ, Jung KJ. A standardization study of Beck Depression Inventory in Korea. J Korean Neuropsychiatr Assoc 1986;25:487-500.

22. Hartka E, Johnstone B, Leino EV, Motoyoshi M, Temple MT, Fillmore KM. A meta-analysis of depressive symptomatology and alcohol consumption over time. $\mathrm{Br} J$ Addict 1991;86:1283-1298.

23. Umene-Nakano W, Yoshimura R, Ikenouchi-Sugita A, Hori $\mathrm{H}$, Hayashi K, Ueda N, et al. Serum levels of brain-derived neurotrophic factor in comorbidity of depression and alcohol dependence. Hum Psychopharmacol 2009;24:409-413.

24. Diniz BS, Teixeira AL, Machado-Vieira R, Talib LL, Gattaz WF, Forlenza OV. Reduced serum nerve growth factor in patients with late-life depression. Am J Geriatr Psychiatry 2013;21:493-496.

25. Pariante $\mathrm{CM}$, Lightman SL. The HPA axis in major depression: classical theories and new developments. Trends Neurosci 2008;31:464-468.

26. Mueller TI, Lavori PW, Keller MB, Swartz A, Warshaw M, Hasin D, et al. Prognostic effect of the variable course of alcoholism on the 10-year course of depression. Am J Psychiatry 1994;151:701-706. 